\section{International Classification of Diseases 10th edition-based disability adjusted life years for measuring of burden of specific injury}

\author{
Yu Jin Kim ${ }^{1}$, Sang Do Shin ${ }^{1,2}$, Hye Sook Park ${ }^{3}$, Kyoung Jun Song ${ }^{2}$, \\ Jin Sung $\mathrm{Cho}^{4}$, Seung Chul Lee ${ }^{5}$, Sung Chun Kim${ }^{6}$, Ju Ok Park', \\ Ki Ok Ahn ${ }^{8}$, Yu Mi Park ${ }^{9}$

\begin{abstract}
'Department of Emergency Medicine, Seoul National University College of Medicine, Seoul, Korea ${ }^{2}$ Department of Emergency Medicine, Seoul National University Hospital, Seoul, Korea ${ }^{3}$ Department of Preventive Medicine, Ewha Womans University College of Medicine, Seoul, Korea ${ }^{4}$ Department of Emergency Medicine, Gachon University Gil Hospital, Incheon, Korea ${ }^{5}$ Department of Emergency Medicine, Dongkuk University Ilsan Hospital, Goyang, Korea ${ }^{6}$ Department of Emergency Medicine, Gyeongsang National University Hospital, Jinju, Korea ${ }^{7}$ Department of Emergency Medicine, Hallym University College of Medicine, Dongtan, Korea ${ }^{8}$ Laboratory of Emergency Medical Services, Seoul National University Hospital Biomedical Research Institue, Seoul, Korea

${ }^{9}$ Hallym University School of Public Health, Chuncheon, Korea
\end{abstract}

Objective We aimed to develop an International Classification of Diseases (ICD) 10th edition injury code-based disability-adjusted life year (DALY) to measure the burden of specific injuries.

Methods Three independent panels used novel methods to score disability weights (DWs) of 130 indicator codes sampled from 1,284 ICD injury codes. The DWs were interpolated into the remaining injury codes $(n=1,154)$ to estimate DWs for all ICD injury codes. The reliability of the estimated DWs was evaluated using the test-retest method. We calculated ICD-DALYs for individual injury episodes using the DWs from the Korean National Hospital Discharge Injury Survey (HDIS, $n=23,160$ of 2004) database and compared them with DALY based on a global burden of disease study (GBD-DALY) regarding validation, correlation, and agreement for 32 injury categories.

Results Using 130 ICD 10th edition injury indicator codes, three panels determined the DWs using the highest reliability (person trade-off 1 , Spearman $r=0.724,0.788$, and 0.875 for the three panel groups). The test-retest results for the reliability were excellent (Spearman $r=0.932$ ) $(P<0.001)$. The HDIS database revealed injury burden (years) as follows: GBD-DALY $(138,548)$, GBD-years of life disabled $(130,481)$, and GBD-years of life lost $(8,117)$ versus ICD-DALY $(262,246)$, ICD-years of life disabled $(255,710)$, and ICD-years of life lost $(6,537)$, respectively. Spearman's correlation coefficient of the DALYs between the two methods was $0.759(P<0.001)$, and the Bland-Altman test displayed an acceptable agreement, with exception of two categories among 32 injury groups.

Conclusion The ICD-DALY was developed to calculate the burden of injury for all injury codes and was validated with the GBD-DALY. The ICD-DALY was higher than the GBD-DALY but showed acceptable agreement.

Keywords Disability adjusted life year; Wounds and injuries; International Classification of Diseases
elSSN: 2383-4625

Received: 14 June 2016

Revised: 18 July 2016

Accepted: 4 September 2016

Correspondence to: Sang Do Shin Department of Emergency Medicine, Seoul National University College of Medicine, 101 Daehak-ro, Jongno-gu, Seoul 03080, Korea

E-mail:sdshin@snu.ac.kr

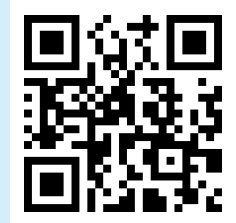

How to cite this article:

Kim YJ, Shin SD, Park HS, Song KJ, Cho JS, Lee SC, Kim SC, Park JO, Ahn KO, Park YM. International Classification of Diseases 10th edition-based disability adjusted life years for measuring of burden of specific injury. Clin Exp Emerg Med 2016;3(4):219-238

This is an Open Access article distributed under the terms of the Creative Commons Attribution Non-Commercial License (http:// creativecommons.org/licenses/by-nc/4.0/). 


What is already known
The disability-adjusted life years measures the burden of disease adjusting for mortality and disability using 33 injury
categories but is too crude.
What is new in the current study
This study shows that International Classification of Disease 10th edition-based injury codes can be reliably used to es-
timate disability-adjusted life years for injuries.

\section{INTRODUCTION}

Injury is the leading cause of death among children and young people, and it is the leading cause of years of potential life lost in most countries.' Injury may also result in disability, which diminishes the subject's quality of life. ${ }^{2}$ The disability-adjusted life year (DALY) was created to measure the burden of disease after adjusting for both mortality and disability, and is a useful method for health policy decision-making. ${ }^{3-6}$ The DALY has been used to evaluate the global burden of disease (GBD) for major diseases, including injury. ${ }^{7-10}$ However, injury is not a single disease entity but a group of very complex processes consisting of multiple injury mechanisms and natures of injury. Previous GBD studies have categorized injury into 32 distinct injury groups." Although the burden of injury may be measured using these simple categories, the GBD of injury may be too crude to reveal the burden of each specific injury. For example, the GBD group classified the burden of poisoning as a single category. However, poisoning involves various materials, ranging from mild substances (i.e., sedatives) to extremely fatal substances (i.e., paraquat). ${ }^{12} \mathrm{~A}$ more specific method for measuring the disability associated with specific injuries would allow researchers and administrators in more precisely assessing subject disability in large databases. The International Classification of Diseases (ICD) includes all injuries, injury subgroups, and adverse effects. The study hypothesis is that developing a method to measure the disability of each injury diagnostic code (ICD 10th edition S/T codes) would allow the calculation of the entire burden of specific injuries. The objectives of the current study were to develop an ICD 10th edition-based disability-adjusted life year (DALY) (ICD-DALY) for injury and to test its reliability and validity.

\section{METHODS}

This study was supported by the Ministry of Health, Welfare, and
Family Affairs of Republic of the Korea in 2008 (Health Promotion Fund A0104208A00). The Seoul National University Hospital institutional review board approved the study with waiver of informed consent because the study did not require the enrollment of human participants. Patient records/information were anonymized and de-identified prior to analysis. The study flow diagram is presented in Fig. 1.

\section{Determining the disability weight for injury codes}

To determine the disability weight (DW) for each injury S/T code ( $n=1,284$ codes), we randomly sampled 10\% ( $n=130$ codes) of the ICD 10th edition (ICD-10) injury codes from an existing injury database (National Injury Database, NIDB) using a stratified random sampling. The NIDB $(n=29,285,528)$ included total injury data for all patients who had utilized medical services between 2001 and 2003 in Korea; 93.7\% for outpatients, 6.0\% for hospital admissions, and $0.3 \%$ for death after treatment. ${ }^{13}$ Using the NIDB, we calculated the admission rate ratio (ARR) as the number of deaths and hospital admissions associated with a specific ICD injury code over the total number of patients corresponding to the same ICD injury code. Stratified by the ARR distribution per 10 percentiles, we randomly sampled the indicator injury codes in even order $(n=130)$ (Appendix 1). As the ARR reflects morbidity and mortality associated with specific injury codes, the 10th percentile of the ARR represents a stratum for which no valid parameter exists.

The DW for each injury code was determined using the same method applied by the GBD research group. ${ }^{11}$ We established three panel groups, each composed of six experts (five emergency and trauma care physicians and one preventive medicine physician) and one trained coordinator. There was no panel turnover and there were no panel dropouts during the one full-day survey workshop for DW measurement. Each panel group was assigned to separate rooms to avoid any bias. The 130 indicator injury codes were randomly assigned to one of the three groups. An ad- 


\section{DW estimation}

Random sampling (10\%) of 130 indicator diagnoses from injury related ICD-10 codes $(1,284)$

DW estimation (130 indicator diagnoses): panel study (VAS, PT01, PT02, TTO)

Interpolation of DW of injury related ICD-10 codes $(1,284)$ through VAS method

Reliability test (test-retest)

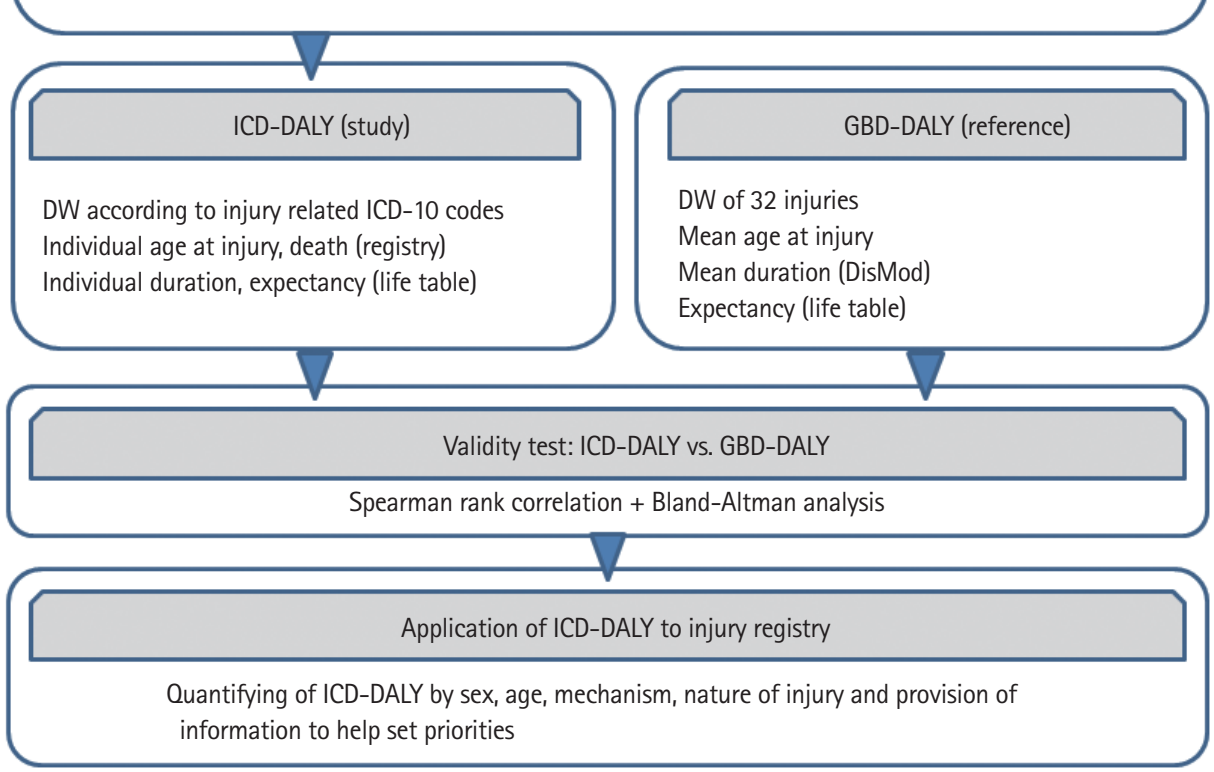

Fig. 1. Study flow diagram. DW, disability weight; ICD-10, International Classification of Diseases 10th edition; VAS, visual analogue scale; PT01, person trade-off 1; PTO2, person trade-off 2; TTO, time trade-off; DALY, disability-adjusted life years; GBD, Global Burden of Disease Study.

ditional 16 codes were shared by the groups to calculate the level of agreement between the groups via correlation analysis. Ideally, all three panels should evaluate all 130 indicator diagnoses independently. However, cost and time limitations existed. Therefore, we assigned a fixed number of codes ( 16 common codes and 38 different codes) per panel with the assumption that approximately 10 minutes were required to determine the DW of each injury code. We benchmarked this method on the basis of previous studies. ${ }^{14,15}$ To determine the DW, we utilized four separate methods, as follows: visual analogue scale (VAS), person trade-off 1 (PT01), person trade-off 2 (PT02), and time trade-off (TTO). These were used to determine the DW in prior studies. ${ }^{16,17}$ For example, in the VAS method, one panel member discloses their VAS result regarding a specific code to the other members in their group and explains their reasoning. After discussion, each panel reconsiders the VAS for each injury code and documents the score on a designed questionnaire. This process was performed and repeated for all injury codes (54 codes) assigned to each panel. The median value for every code assessed using each method was calculated and considered to be the DW score. After calculating the DW score, we selected the most reliable of the four methods (VAS, PT01, PTO2, or TT0) by calculating Spearman's correlation for the 16 common codes assigned to the three panel groups.

Upon determining the DW for each indicator injury code $(n=130)$, we estimated the DW for the remaining injury codes using the interpolation method. ${ }^{18}$ Each panel scored the DW for the remaining 1,154 S/T codes (i.e., 385 codes for each of the three panel groups). The panels referenced the DW values of the 130 indicator injury codes. For this procedure, the panels used the VAS method, as it required the least amount of time to generate an agreement. After scoring the DW for all injury codes, the median VAS among the panels was calculated to determine the DW for each injury code. Finally, we estimated the DWs for all 1,284 ICD-10 injury codes.

To determine the reliability of the DW of each code, we used the test-retest method for the 16 common indicator injury codes. Two months after completing the initial panel study, we repeated the same panel study using the same procedural methods for VAS, PT01, PT02, and TTO. Pearson's correlation analysis was used to assess the reliability of the test-retest method. 


\section{Calculating the ICD-DALY using the estimated DW of in- jury codes}

To calculate the DALY, we used the same formula, which has been proposed in several previous studies., ${ }^{2,3}$ The ICD-DALY was calculated using the same formula as the conventional DALY (GBDDALY), but the specific DW of the ICD codes and assumptions regarding duration of morbidity and life expectancy differed. We considered the discount rate $(\gamma=0.03)$, age weight parameter $(\beta=0.04)$, modulation factor ( $K=0$ or 1$)$ according to age weight, and constant $(C=0.1658)$ to calculate the DALY for each injury episode. The estimated life expectancy ("L" in years of life lost [YLL]) or duration of morbidity due to a specific injury ("L" in years of life disabled [YLD]) was calculated from the age at the time of the injury event to the life expectancy, based on the life tables of the National Statistical Office of Korea. According to previous GBD studies, the cure rate of injury was $0 \%$ for the YLD measurement, and we surrogated the remaining life expectancy for morbidity due to a specific injury. ${ }^{19,20}$ A comparison of essential variables of the ICD-DALY and GBD-DALY calculations are shown in Appendix 2.

\section{Validation of the ICD-DALY}

To validate the ICD-DALY, we compared it with the conventional GBD-DALY. We assessed and compared both DALY results (ICDDALY vs. GBD-DALY) using a pre-existing injury database, the Korean National Hospital Discharge Injury Survey. The Hospital Discharge Injury Survey (HDIS) is a nationwide, stratified sampled, and abstract survey data from 170 general hospitals with more than 100 beds in Korea since 2004 by Korea Centers for Disease Control and Prevention, including all patients with diagnosis code

Table 1. Indicator injury ICD-10 codes sampled from total injury ICD-10 codes using the admission rate ratio

\begin{tabular}{ccc}
\hline $\begin{array}{c}\text { Admission } \\
\text { rate ratio }\end{array}$ & $\begin{array}{c}\text { Injury ICD-10 codes, } \\
\text { total }\end{array}$ & $\begin{array}{c}\text { Indicator injury ICD-10 } \\
\text { codes sampled }\end{array}$ \\
\hline Total & $1,284(100.0)$ & $130(100.0)$ \\
$0.00-0.05$ & $181(14.1)$ & $18(13.8)$ \\
$0.05-0.15$ & $295(23.0)$ & $30(23.1)$ \\
$0.15-0.25$ & $208(16.2)$ & $21(16.2)$ \\
$0.25-0.35$ & $146(11.4)$ & $15(11.5)$ \\
$0.35-0.45$ & $113(8.8)$ & $11(8.5)$ \\
$0.45-0.55$ & $108(8.4)$ & $11(8.5)$ \\
$0.55-0.65$ & $73(5.7)$ & $7(5.4)$ \\
$0.65-0.75$ & $75(5.8)$ & $8(6.2)$ \\
$0.75-0.85$ & $47(3.7)$ & $5(3.8)$ \\
$0.85-0.95$ & $32(2.5)$ & $3(2.3)$ \\
$0.95-1.00$ & $6(0.5)$ & $1(0.8)$ \\
\hline
\end{tabular}

Values are presented as number (\%).

ICD-10, International Classification of Diseases 10th edition.
(ICD S or T codes) at discharge hospital. We recategorized 1,284 injury-related ICD codes into 32 categories using the "GBD 2000 nature of injury categories and ICD codes" (http://www.who.int/ healthinfo/statistics/bod_injuries.pdf). We calculated the GBDDALY by using the mean age of injury event, mean duration of injury prevalence (YLD) or mean life expectancy (YLL) using DisMod II (http://www.who.int/healthinfo/global_burden_disease/tools_ software/en/). The DisMod II is a software tool that can be used to verify the consistency of estimates of incidence, prevalence, duration, and case fatality for diseases. We estimated the incidence and case fatality for 32 categories of injury, by sex and age group, and replaced the remission rate with $0 \%$, as previously reported. ${ }^{21,22}$ We calculated the ICD-DALY using individual age at the time of injury, individual age at death, and the estimated life expectancy based on the life tables of the National Statistical Office of Korea and ICD-10 code-based DW. Spearman's rank correlation test and the Bland-Altman test for agreement were used to compare 32 injury categories between ICD-DALY and GBD-DALY.

\section{RESULTS}

\section{DW estimations and the calculation of the ICD-DALY}

We sampled 130 indicator ICD-10 injury codes based on the distribution of the ARR of each ICD-10 code in the NIDB. Appendix 1 lists the 130 selected indicator ICD-10 injury codes. Table 1 displays the distribution injury codes according to the ARR. The 130 codes were distributed similarly according to the strata of the ARR percentile. Table 2 displays Spearman's rank correlation coefficients among the panel groups for each of the four methods (i.e., VAS, PT01, PT02, and TTO). The PT01 method demonstrated the highest correlation coefficient among the three panel groups $(0.788,0.685$, and 0.875$)$. Fig. 2 shows the distribution of the median DWs for the 16 common indicator injury codes derived from a panel study applying four different methods. The values derived using the VAS and PT01 methods were distributed evenly throughout the total range of 0.0 to 1.0 , while the values derived using the PTO2 and TTO methods were densely concentrated below 0.2. Therefore, we selected the DW for 160 indicator ICD in-

Table 2. Spearman rank correlation coefficients between two panels according to valuation methods

\begin{tabular}{lccc}
\hline Valuation method & Panel 1-2 & Panel 2-3 & Panel 1-3 \\
\hline VAS & $0.686(P=0.003)$ & $0.708(P=0.002)$ & $0.690(P=0.003)$ \\
PT01 & $0.788(P=0.003)$ & $0.685(P=0.003)$ & $0.875(P=0.001)$ \\
PT02 & $0.549(P=0.028)$ & $0.685(P=0.003)$ & $0.754(P=0.007)$ \\
TO & $0.403(P=0.014)$ & $0.510(P=0.052)$ & $0.723(P=0.023)$ \\
\hline
\end{tabular}

VAS, visual analogue scale; PT01, person trade-off $1 ;$ PTO2, person trade-off $2 ;$ TTO, time trade-off. 
jury codes derived using the PTO1 method, which was considered the most reliable and discriminative method for determining DW. We determined the DW of the remaining ICD-10 injury codes using the interpolation method. Fig. 3 shows the markedly high correlation between the VAS- and PT01-based DWs for the 130 indicator ICD-10 injury codes $(\rho=0.721, P<0.001)$. Using the VASbased interpolation method, we calculated the median values of the DWs for each ICD-10 code. Appendix 3 displays the DWs of all injury codes.

We performed a test-retest study on the 16 common indicator ICD-10 injury codes. Pearson's correlation coefficient and Spearman's rank correlation coefficient between the test-retest of DW valuation via the PT01 method were $0.932(P<0.001)$ and 0.740 $(P<0.001)$, respectively, thereby demonstrating good reliability (Table 3). Pearson's correlation coefficients between the test-retest results of the 5 panelists participating in the study were as

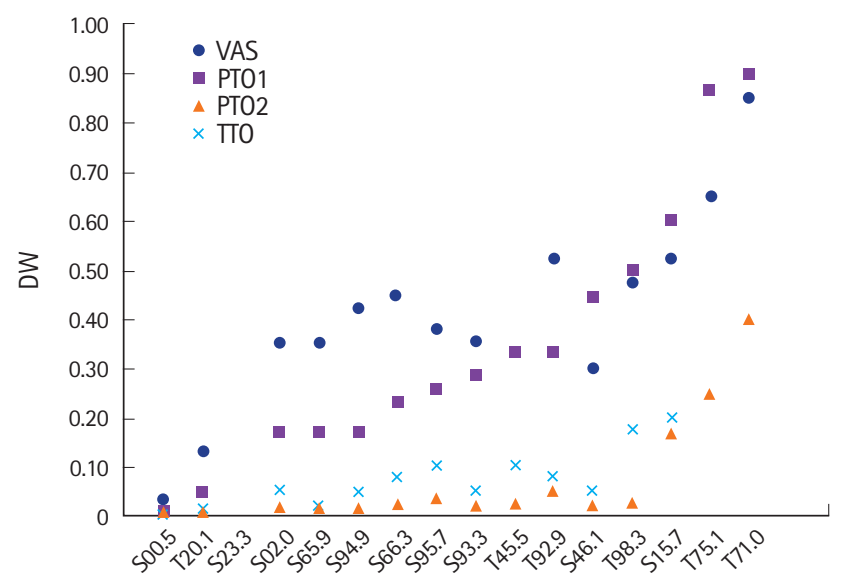

Fig. 2. Distributions of median disability weight (DW) calculated by each panel method. VAS, visual analogue scale; PTO1, person trade-off 1; PTO2, person trade-off 2; TTO, time trade-off.

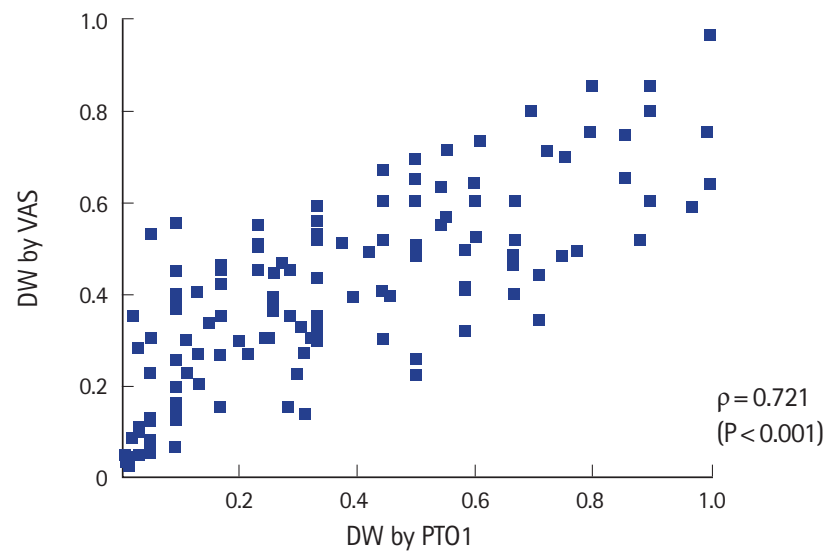

Fig. 3. Correlation between person trade-off 1 (PT01)-based and visual analogue scale (VAS)-based disability weights (DWs) for 130 indicator codes. follows: $0.728,0.852,0.891,0.937$, and 0.962 (all $P<0.001$ ). The formula was completed to calculate the ICD-DALY using estimated DWs and variables in an existing injury database.

\section{Validation of the ICD-DALY}

Most codes in the HDIS $(n=23,160$, collected in 2004, male subjects $61.5 \%$, adults ( 15 to 64 years old) $77.3 \%$ and elderly ( $\geq 65$ years old) $17.9 \%$ were automatically converted into 32 nature of injury categories using the GBD-DALY method, including 271 codes by manual conversion by the investigators (conversion rate $1,166 / 1,284=91 \%)$. However, 118 codes were not reclassified into 32 injury categories due to: extremely rare nature of the injury, non-traumatic injury, post-injury complication, environmental injury, or side effects of medical treatment. The excluded codes are displayed in Appendix 4.

Table 4 displays the GBD-DALY and ICD-DALY results according to 32 categories derived from the HDIS database. The GBD-DALY, GBD-YLD, and GBD-YLL were 138,548, 130,481, and 8,117 years, respectively. The ICD-DALY, ICD-YLD, and ICD-YLL were 262,246, 255,710, and 6,537 years, respectively. The mean YLD/YLL proportions were 16.1 for the GBD-DALY and 39.1 for the ICD-DALY.

Fig. 4 shows the comparison between the ICD-DALY and the GBD-DALY based on 32 nature of injury categories. The injury distribution differed between the GBD-DALY and the ICD-DALY (e.g., intracranial injuries $>$ sprains $>$ fracture-face bones $>$ open wound > fracture-patella, tibia, or fibula > poisoning for GBD-DA-

Table 3. Test-retest results of 16 common indicator ICD-10 injury codes using person trade-off 1 (median)

\begin{tabular}{lcc}
\hline ICD-10 code & Test & Retest \\
\hline S00.5 & 0.005 & 0.004 \\
S02.0 & 0.200 & 0.167 \\
S15.7 & 0.375 & 0.375 \\
S23.3 & 0.074 & 0.034 \\
S46.1 & 0.167 & 0.200 \\
S65.9 & 0.167 & 0.153 \\
S66.3 & 0.167 & 0.167 \\
S93.3 & 0.167 & 0.153 \\
S94.9 & 0.167 & 0.138 \\
S95.7 & 0.231 & 0.167 \\
T20.1 & 0.029 & 0.015 \\
T45.5 & 0.200 & 0.130 \\
T71.0 & 0.714 & 0.714 \\
T75.1 & 0.800 & 0.833 \\
T92.9 & 0.310 & 0.091 \\
T98.3 & 0.444 & 0.167 \\
\hline
\end{tabular}

Pearson correlation coefficient $=0.932(\mathrm{P}<0.001)$; Spearman correlation coefficients $=0.740(P<0.001)$.

ICD-10, International Classification of Diseases 10th edition. 
Table 4. Comparison of GBD-DALY and ICD-DALY according to 32 nature of injury categories

\begin{tabular}{|c|c|c|c|c|c|c|c|c|}
\hline Nature of injury category & GBD-YLD (a) & GBD- YLL (b) & $(a) /(b)$ & GBD- DALY & ICD-YLD (c) & ICD- YLL (d) & $(c) /(d)$ & ICD- DALY \\
\hline Fracture-skull & 3,990 & 603 & 6.6 & 4,593 & 6,530 & 361 & 18.1 & 6,891 \\
\hline Fracture-face bones & 11,367 & 281 & 40.5 & 11,647 & 10,854 & 63 & 172.3 & 10,917 \\
\hline Fracture-rib or sternum & 2,955 & 341 & 8.7 & 3,296 & 6,974 & 215 & 32.4 & 7,189 \\
\hline Fracture-pelvis & 1,901 & 180 & 10.6 & 2,081 & 4,174 & 112 & 37.3 & 4,286 \\
\hline Fracture-clavicle, scapula, or humerus & 3,579 & 199 & 18.0 & 3,778 & 8,149 & 55 & 148.2 & 8,204 \\
\hline Fracture-hand bones & 1,987 & 10 & 198.7 & 1,997 & 4,873 & 3 & $1,624.3$ & 4,876 \\
\hline Fracture-femur & 4,577 & 280 & 16.3 & 4,857 & 6,824 & 159 & 42.9 & 6,983 \\
\hline Fracture-patella, tibia, or fibula & 8,158 & 126 & 64.7 & 8,284 & 13,213 & 54 & 244.7 & 13,267 \\
\hline Fracture-ankle & 1,101 & 0 & NA & 1,101 & 2,096 & 0 & NA & 2,096 \\
\hline Fracture-foot bones & 1,112 & 0 & NA & 1,112 & 4,734 & 0 & NA & 4,734 \\
\hline Injured spinal cord & 3,658 & 72 & 50.8 & 3,730 & 2,519 & 36 & 70.0 & 2,555 \\
\hline Intracranial injuries & 24,177 & 2,342 & 10.3 & 26,519 & 36,465 & 2,252 & 16.2 & 38,716 \\
\hline Internal injuries & 3,285 & 1,259 & 2.6 & 4,543 & 16,176 & 1,373 & 11.8 & 17,549 \\
\hline Open wound & 8,808 & 459 & 19.2 & 9,267 & 13,157 & 89 & 147.8 & 13,246 \\
\hline Injury to eyes & 2,558 & 57 & 44.9 & 2,615 & 5,194 & 12 & 432.8 & 5,206 \\
\hline Amputations-thumb & 240 & 0 & NA & 240 & 780 & 0 & NA & 780 \\
\hline Amputations-finger & 615 & 0 & NA & 615 & 3,021 & 0 & NA & 3,021 \\
\hline Amputations-arm & 328 & 0 & NA & 328 & 486 & 0 & NA & 486 \\
\hline Amputations-toe & 10 & 0 & NA & 10 & 46 & 0 & NA & 46 \\
\hline Amputations-foot & 18 & 0 & NA & 18 & 37 & 0 & NA & 37 \\
\hline Amputations-leg & 157 & 0 & NA & 157 & 224 & 0 & NA & 224 \\
\hline
\end{tabular}

GBD, Global Burden of Disease Study; DALY, disability-adjusted life year; ICD, International Classification of Diseases 10th edition; YLD, years of life disabled; YLL, years of life lost; NA, not applicable.

LY and sprains $>$ intracranial injuries $>$ dislocation-other dislocation $>$ internal injuries $>$ fracture-patella, tibia, or fibula $>$ open wound for ICD-DALY). The ICD-DALY data exceeded the GBD-DALY results in most injury categories. The ICD-DALY results displayed an increased burden compared with the GBD-DALY results by 18 -fold, for the burns less than $20 \%$ category, and by 6 -fold, for the burns greater than 60\% category. Meanwhile, the ICDDALY was lower for the fracture-face bones and spinal cord injury categories compared with the GBD-DALY.

We observed a high correlation between the GBD-DALY and ICD-DALY. Spearman's rank correlation coefficients for YLL, YLD, and DALY were $0.988(P<0.001), 0.738(P<0.001)$, and 0.759 $(P<0.001)$, respectively (Fig. $5 A-C)$. To test the agreement be- tween the two methods, we analyzed the results using the BlandAltman test (Fig. 5D). Two categories were in disagreement, thereby demonstrating the limits of the agreement range (e.g., dislocation-other dislocation and sprains).

\section{DISCUSSION}

We developed the ICD-DALY in the current study, which represents the first attempt to describe an injury measurement for specific injury codes regarding the GBD. Most GBD studies have

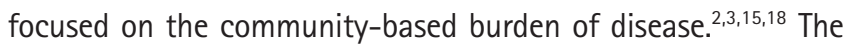
GBD-DALY has potential for comparing the burden of disease but not individuals with specific injuries. However, the current study 
Yu Jin Kim, et al.

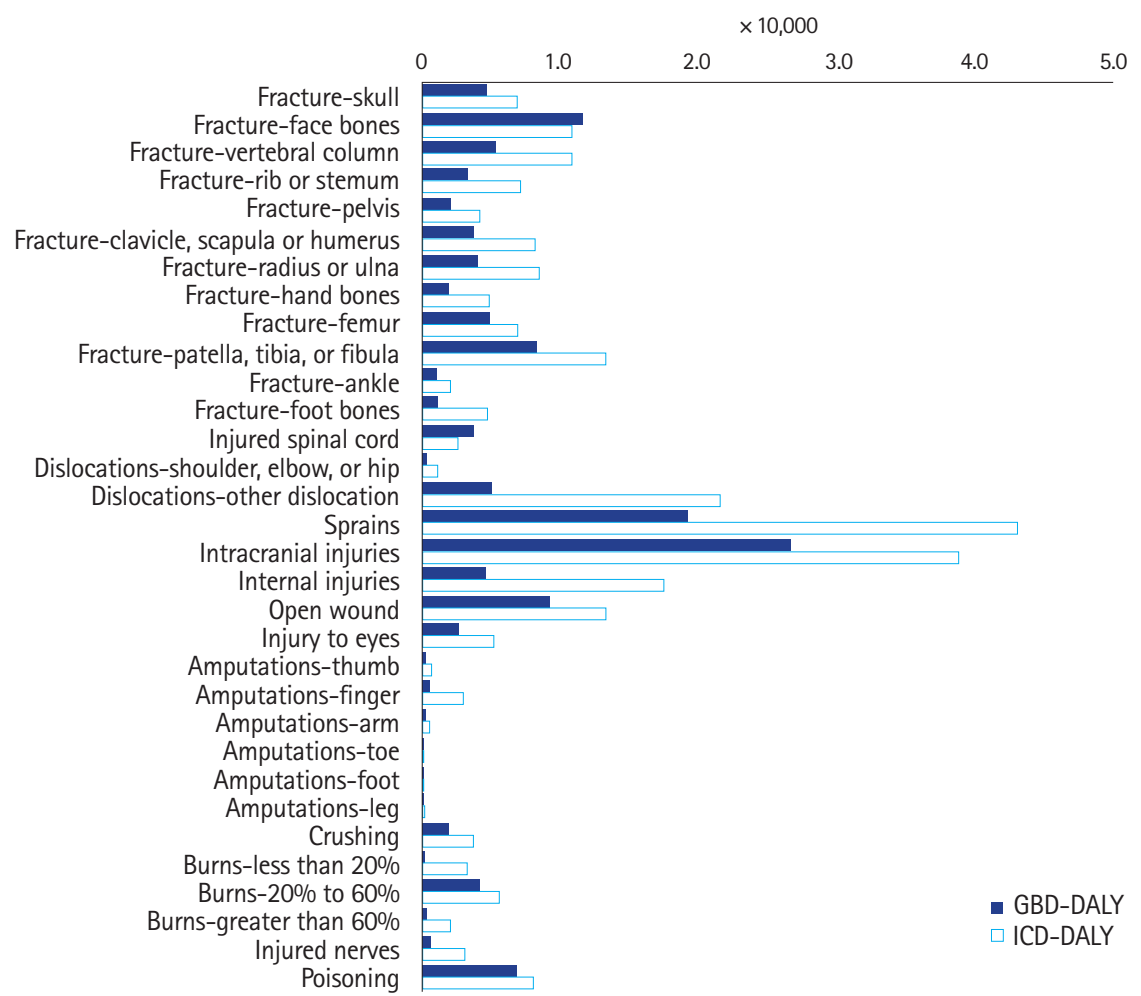

Fig. 4. Comparison of global burden of disease (GBD)-disability-adjusted life year (DALY) and International Classification of Disease 10th edition (ICD)DALY by 32 injury nature categories.
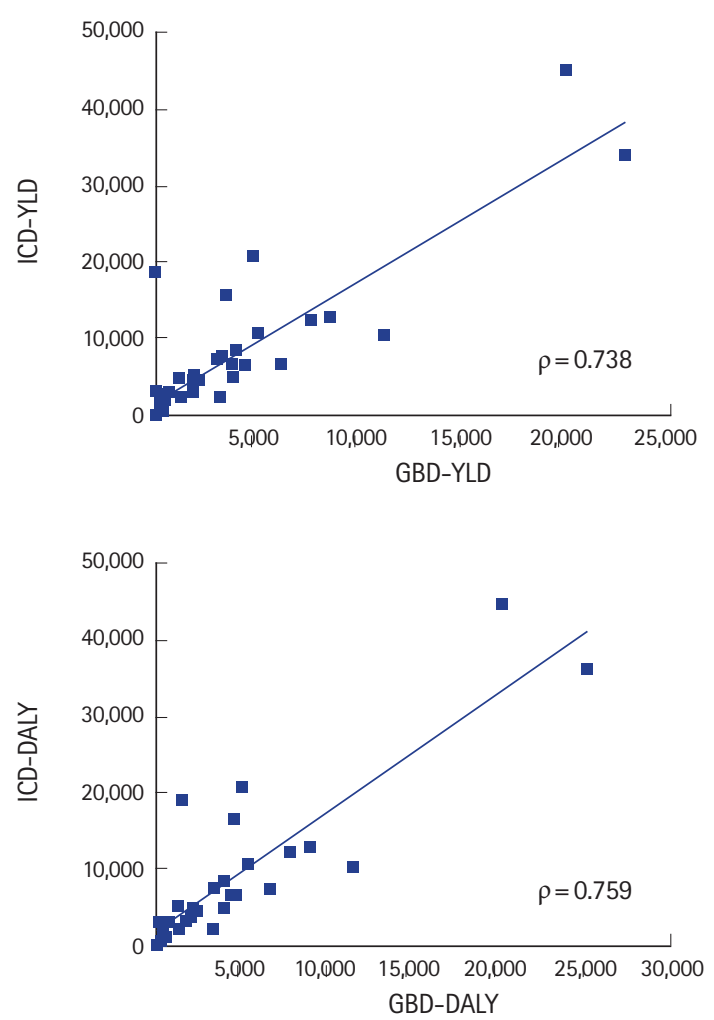

(A)

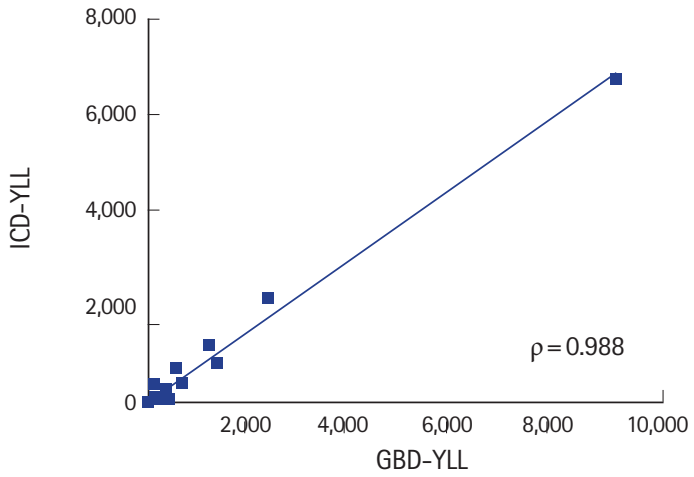

B

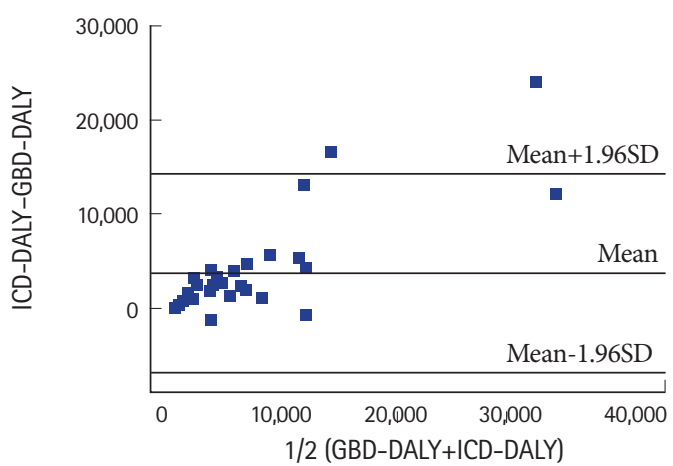

Fig. 5. Comparison of global burden of disease (GBD)-disability-adjusted life year (DALY) and International Classification of Disease 10th edition (ICD)DALY. (A) Comparison for years of life disabled (YLD), (B) comparison for years of life lost (YLL), (C) comparison for DALY, and (D) Bland-Altman plot. SD, standard deviation. 
focused on individual data with specific individual injury diagnosis codes. In many countries, injury surveillance and nation-wide injury datasets exist, which include exact diagnosis codes and injury mechanisms. Most injury datasets include subject age, diagnosis, time of injury, and mortality outcome. These variables are included for analyzing risk factors, developing injury prevention strategies, and evaluating the effect of such interventions. Using this tool and these three variables, the ICD-DALY can be calculated for every injured individual.

The current technique described in our study is a cost-saving method designed for calculating the burden of injury. Resource intensive methods, including the Disability Rating Scale and the Glasgow Outcome Scale, assess the individual disability of an injured victim, but these tools require follow-up interviews with the subject. ${ }^{21,22}$ Although these clinical tools provide a reliable assessment of patient disability, their limitations primarily involve the amount of effort required to perform the calculations. In contrast, the ICD-DALY provides a risk-based DALY, which requires fewer resources and is applicable across large databases. This tool now allows for these new outcome parameters in injury research, incidence, mortality, and DALY per individual patient. Hospitalbased injury data are also useful for calculating disability and are comparable with other conditions. ${ }^{23}$

This study used a complex methodology to determine the DW derived from injury diagnostic codes. Injury codes vary in terms of severity, mechanism, and outcome, but an injury group cannot be considered one disease entity. Poisonings display a broad spectrum of severity; therefore, a DW for poisoning must account for specific subcategories. Therefore, the determination of a disability for each individual ICD-10 injury code is ideal, but there are too many injury codes $(n=1,284)$ to be decided via expert-based consensus (panel study). Therefore, indicator injury codes $(n=130$, $10 \%$ ) based on the strata by morbidity/mortality (like ARR) were chosen. To interpolate the DW using the value of the indicator ICD-10 codes, it is essential to have evenly distributed DW values for all ICD-10 codes. We successfully selected indicator injury codes and then interpolated the corresponding DWs into the remaining ICD-10 injury codes. Previous panel studies have demonstrated varying methodological results. ${ }^{16,17,24}$ In accordance with previous studies, the current study demonstrated that the PTO1 method displays the best correlation among panel groups. We accepted the PT01 method as the ideal tool and used it to determine the DWs for the 130 codes, which were then interpolated in the next step. The PTO1 and VAS methods demonstrated a high correlation for common standard injury codes. We used the VAS method for interpolation to all other codes, which demonstrated a good correlation performance with the PT01 method. Similarly, other studies have utilized the VAS method due to this benefit. ${ }^{15,18}$ This study tested the reliability of the DW of each code using the test-retest method. ${ }^{25}$ We found excellent reliability among the median DWs derived from the PT01 method.

Validation was performed by comparing the ICD-DALY and GBD-DALY values of 32 nature of injury categories, as a direct comparison for all injury codes was impossible. Both measurements correlated strongly $(\rho=0.759, P<0.001)$, although the ICD-DALY were higher than the GBD-DALY results across most categories. The Bland-Altman test showed an acceptable agreement in most categories, with the exception of two categories. Overall, higher agreements were observed between the ICD-DALY and GBD-DALY results in homogeneous categories with similar anatomic areas, injury depth, and severity. Meanwhile, for the remaining heterogeneous categories with different anatomical injuries, the agreement was poor between ICD-DALY and GBD-DALY (e.g., dislocation-shoulder/elbow/hip, dislocation-other dislocation, sprain, intracranial injury, internal injury, open wound, injury of the eyes, crushing, burns-less than 20\%, burns-20\% to $60 \%$, burns-greater than 60\%, injured nerves and poisoning). We assume that the differences between ICD-DALY and GBD-DALY are more marked in the heterogeneous injury categories because they have an increased variety of ICD injury codes compared with the homogeneous category. The GBD-DALY may have excessively reclassified all injuries into 32 injury categories. For example, neurologic deficits, soft tissue injuries, burns, and poisoning would be overlooked in 32 injury categories despite the wide range of disabilities that these injuries incur. The GBD-YLL and ICD-YLL showed very good agreement but GBD-YLD and ICD-YLD showed poorer agreement (Fig. 5A, B). The YLD can be calculated from death, which is clearly defined, for calculation but YLD might be incorrect due to different DWs of injury categories.

This study has certain limitations. First, the interpolation method may be used to determine the DALY. Only 10\% of the indicator injury codes were reviewed by the panel groups, while the other codes were estimated via interpolation. Although we tested the reliability of the DW as an indicator for the ICD code $(n=16)$, the entire ICD-10 code was not tested for reliability. Second, we calculated DALYs using the maximum DW among the multiple injury codes that were diagnosed during the same episode (multiple injuries). Therefore, the DALYs might be underestimated because the remaining injury-associated disability was not included. For patients with multiple injuries, the DALY would be minimally calculated. Third, our validation method in which two DALY results were compared is limited in terms of the injury codes that were not compared due to the difficulty of re-categorizing the injury codes into 32 injury categories. Finally, the ICD-DALY method was 
not validated with clinical disability parameters or the qualityadjusted life year. ${ }^{26}$

To efficiently calculate the burden of injury for individual victims, the ICD-10-based DALY was developed and validated using a nationwide database. We found that this new method was easy and feasible for estimating the disability of each individual injury victim and comparable to the GBD-DALY. The ICD-DALY should be extensively validated to apply it for injury epidemiology and prevention.

\section{CONFLICT OF INTEREST}

No potential conflict of interest relevant to this article was reported.

\section{ACKNOWLEDGMENTS}

This study was supported by the Ministry of Health, Welfare, and Family Affairs of Republic of the Korea in 2008 (Health Promotion Fund A0104208A00).

\section{REFERENCES}

1. Murray $C J$, Lopez AD, Jamison DT. The global burden of disease in 1990: summary results, sensitivity analysis and future directions. Bull World Health Organ 1994;72:495-509.

2. Murray CJ, Lopez AD. Mortality by cause for eight regions of the world: Global Burden of Disease Study. Lancet 1997;349: 1269-76.

3. Murray CJ, Lopez AD. Global mortality, disability, and the contribution of risk factors: Global Burden of Disease Study. Lancet 1997;349:1436-42.

4. Lopez AD, Mathers CD, Ezzati M, Jamison DT, Murray CJ. Global and regional burden of disease and risk factors, 2001: systematic analysis of population health data. Lancet 2006; 367:1747-57.

5. Murray CJ, Lopez AD, Wibulpolprasert S. Monitoring global health: time for new solutions. BMJ 2004;329:1096-100.

6. Fischer Walker CL, Ezzati M, Black RE. Global and regional child mortality and burden of disease attributable to zinc deficiency. Eur J Clin Nutr 2009;63:591-7.

7. Hogan P, Dall T, Nikolov P; American Diabetes Association. Economic costs of diabetes in the US in 2002. Diabetes Care 2003;26:917-32.

8. Vlajinac H, Sipetic S, Saulic A, Atanackovic Z, Marinkovic J, Bjegovic V. Burden of ischaemic heart disease and cerebrovascular diseases in Serbia without Kosovo and Metohia, 2000.
Eur J Cardiovasc Prev Rehabil 2006;13:753-9.

9. Ustun TB, Ayuso-Mateos JL, Chatterji S, Mathers C, Murray CJ. Global burden of depressive disorders in the year 2000. $\mathrm{Br}$ J Psychiatry 2004;184:386-92.

10. Michaud CM, McKenna MT, Begg S, et al. The burden of disease and injury in the United States 1996. Popul Health Metr 2006;4:11.

11. Murray CJ, Lopez AD. The global burden of disease: a comprehensive assessment of mortality and disability from diseases, injuries, and risk factors in 1990 and projected to 2020. Cambridge, MA: Harvard University Press; 1996.

12. Jelsma J, De Weerdt W, De Cock P. Disability adjusted life years (DALYs) and rehabilitation. Disabil Rehabil 2002;24: 378-82.

13. Kim J, Shin SD, Im TH, et al. Development and validation of the excess mortality ratio-adjusted injury severity score using the International Classification of Diseases 10th edition. Acad Emerg Med 2009;16:454-64.

14. Yoon SJ, Bae SC, Lee SI, Chang H, Jo HS, Sung JH, et al. Measuring the burden of disease in Korea. J Korean Med Sci 2007; 22:518-23.

15. Yoon SJ, Lee H, Shin Y, Kim Yl, Kim CY, Chang H. Estimation of the burden of major cancers in Korea. J Korean Med Sci 2002; 17:604-10.

16. Essink-Bot ML, Pereira J, Packer C, Schwarzinger M, Burstrom K. Cross-national comparability of burden of disease estimates: the European Disability Weights Project. Bull World Health Organ 2002;80:644-52.

17. Haagsma JA, Polinder S, van Beeck EF, Mulder S, Bonsel GJ. Alternative approaches to derive disability weights in injuries: do they make a difference? Qual Life Res 2009;18:657-65.

18. Melse JM, Essink-Bot ML, Kramers PG, Hoeymans N. A national burden of disease calculation: Dutch disability-adjusted life-years. Dutch Burden of Disease Group. Am J Public Health 2000;90:1241-7.

19. Mathers CD, Vos ET, Stevenson CE, Begg SJ. The burden of disease and injury in Australia. Bull World Health Organ 2001; 79:1076-84.

20. Mathers CD, Vos ET, Stevenson CE, Begg SJ. The Australian Burden of Disease Study: measuring the loss of health from diseases, injuries and risk factors. Med J Aust 2000;172:5926.

21. Hammond FM, Grattan KD, Sasser H, Corrigan JD, Bushnik T, Zafonte RD. Long-term recovery course after traumatic brain injury: a comparison of the functional independence measure and disability rating scale. J Head Trauma Rehabil 2001;16: 318-29. 
22. Struchen MA, Hannay HJ, Contant CF, Robertson CS. The relation between acute physiological variables and outcome on the Glasgow Outcome Scale and Disability Rating Scale following severe traumatic brain injury. J Neurotrauma 2001; 18:115-25.

23. Holtslag $H R$, van Beeck $E F$, Lichtveld RA, Leenen LP, Lindeman $E_{\text {, van }}$ der Werken $C$. Individual and population burdens of major trauma in the Netherlands. Bull World Health Organ 2008;86:111-7.

24. Ubel PA, Loewenstein G, Scanlon D, Kamlet M. Value measurement in cost-utility analysis: explaining the discrepancy between rating scale and person trade-off elicitations. Health Policy 1998;43:33-44.
25. Janssens L, Gorter JW, Ketelaar M, Kramer WL, Holtslag HR. Health-related quality-of-life measures for long-term followup in children after major trauma. Qual Life Res 2008;17:70113.

26. Haagsma JA, van Beeck EF, Polinder S, Hoeymans N, Mulder S, Bonsel GJ. Novel empirical disability weights to assess the burden of non-fatal injury. Inj Prev 2008;14:5-10. 
Appendix 1. One hundred and thirty indicator injury diagnosis

\begin{tabular}{|c|c|c|}
\hline No. & ICD-10 & Diagnosis \\
\hline 1 & S00.5 & Superficial injury of lip and oral cavity \\
\hline 2 & S01.3 & Open wound of ear \\
\hline 3 & S02.0 & Fracture of vault of skull \\
\hline 4 & S02.4 & Fracture of malar and maxillary bones \\
\hline 5 & S02.8 & Fracture of other skull and facial bones \\
\hline 6 & S03.1 & Dislocation of septal cartilage of nose \\
\hline 7 & S05.0 & Injury of conjunctiva and corneal abrasion without mention of foreign body \\
\hline 8 & S05.5 & Penetrating wound of eyeball with foreign body \\
\hline 9 & S05.7 & Avulsion of eye \\
\hline 10 & S10.9 & Superficial injury of neck, part unspecified \\
\hline 11 & S14.3 & Injury of brachial plexus \\
\hline 12 & S14.4 & Injury of peripheral nerves of neck \\
\hline 13 & S15.7 & Injury of multiple blood vessels at neck level \\
\hline 14 & S15.9 & Injury of unspecified blood vessels at neck level \\
\hline 15 & S20.0 & Contusion of breast \\
\hline 16 & S20.4 & Other superficial injuries of back wall of thorax \\
\hline 17 & S20.8 & Superficial injury of other and unspecified parts of thorax \\
\hline 18 & S22.4 & Multiple fracture of ribs \\
\hline 19 & S22.8 & Fracture of other parts of bony thorax \\
\hline 20 & S23.3 & Sprain and strain of thoracic spine \\
\hline 21 & S24.3 & Injury of peripheral nerves of thorax \\
\hline 22 & S25.1 & Injury of innominate or subclavian artery \\
\hline 23 & S27.6 & Injury of pleura \\
\hline 24 & S30.2 & Contusion of external genital organs \\
\hline 25 & S33.0 & Traumatic rupture of lumbar intervertebral disc \\
\hline 26 & S34.8 & Injury of other and unspecified nerves at abdomen, lower back and pelvis level \\
\hline 27 & S38.2 & Traumatic amputation of external genital organs \\
\hline 28 & S40.7 & Multiple superficial injuries of shoulder and upper arm \\
\hline 29 & S43.4 & Sprain and strain of shoulder joint \\
\hline 30 & S46.1 & Injury of muscle and tendon of long head of biceps \\
\hline 31 & S52.2 & Fracture of shaft of ulna \\
\hline 32 & S52.5 & Fracture of lower end of radius \\
\hline 33 & S54.0 & Injury of ulnar nerve at forearm level \\
\hline 34 & S56.2 & Injury of other flexor muscle and tendon at forearm level \\
\hline 35 & S57.8 & Crushing injury of other parts of forearm \\
\hline 36 & S60.0 & Contusion of finger(s) without damage to nail \\
\hline 37 & S62.0 & Fracture of navicular [scaphoid] bone of hand \\
\hline 38 & S63.1 & Dislocation of finger \\
\hline 39 & S63.2 & Multiple dislocation of fingers \\
\hline 40 & S63.7 & Sprain and strain of other and unspecified parts of hand \\
\hline 41 & S65.9 & Injury of unspecified blood vessel at wrist and hand level \\
\hline 42 & S66.3 & Injury of extensor muscle and tendon of other finger at wrist and hand level \\
\hline 43 & S66.5 & Injury of intrinsic muscle and tendon of other finger at wrist and hand level \\
\hline 44 & S68.3 & Combined traumatic amputation of (part of) finger(s) with other parts of wrist and hand \\
\hline 45 & S69.0 & Other and unspecified injuries of wrist and hand \\
\hline 46 & S69.8 & Other specified injuries of wrist and hand \\
\hline 47 & S70.0 & Contusion of hip \\
\hline 48 & S72.9 & Fracture of femur, part unspecified \\
\hline 49 & S74.0 & Injury of sciatic nerve at hip and thigh level \\
\hline 50 & S75.2 & Injury of greater saphenous vein at hip and thigh level \\
\hline 51 & S77.0 & Crushing injury of hip \\
\hline
\end{tabular}

(Continued to the next page) 
Appendix 1. Continued

\begin{tabular}{|c|c|c|}
\hline No. & ICD-10 & Diagnosis \\
\hline 52 & S80.9 & Superficial injury of lower leg, unspecified \\
\hline 53 & S82.3 & Fracture of lower end of tibia \\
\hline 54 & S83.2 & Tear of meniscus, current \\
\hline 55 & S84.0 & Injury of tibial nerve at lower leg level \\
\hline 56 & S88.9 & Traumatic amputation of lower leg, level unspecified \\
\hline 57 & S89.9 & Unspecified injury of lower leg \\
\hline 58 & S92.5 & Fracture of other toe \\
\hline 59 & S93.3 & Dislocation of other and unspecified parts of foot \\
\hline 60 & S94.9 & Injury of unspecified nerves at ankle and foot level \\
\hline 61 & S95.7 & Injury of multiple blood vessels at ankle and foot level \\
\hline 62 & T01.2 & Open wounds involving multiple regions of upper limb(s) \\
\hline 63 & T01.6 & Open wounds involving multiple regions of upper limb(s) with lower limb(s) \\
\hline 64 & T04.3 & Crushing injuries involving multiple regions of lower limb(s) \\
\hline 65 & T05.6 & Traumatic amputation of upper and lower limbs, any combination[any level] \\
\hline 66 & T05.9 & Multiple traumatic amputations, unspecified \\
\hline 67 & T06.3 & Injuries of blood vessels involving multiple body regions \\
\hline 68 & T09.0 & Superficial injury of trunk, level unspecified \\
\hline 69 & T09.6 & Traumatic amputation of trunk, level unspecified \\
\hline 70 & T16.0 & Foreign body in ear \\
\hline 71 & T17.8 & Foreign body in other and multiple parts of respiratory tract \\
\hline 72 & $\mathrm{~T} 20.1$ & Burn of first degree of head and neck \\
\hline 73 & T21.3 & Burn of third degree of trunk \\
\hline 74 & $\mathrm{~T} 24.3$ & Burn of third degree of hip and lower limb, except ankle and foot \\
\hline 75 & T25.3 & Burn of third degree of ankle and foot \\
\hline 76 & $\mathrm{~T} 25.7$ & Corrosion of third degree of ankle and foot \\
\hline 77 & $\mathrm{~T} 26.2$ & Burn with resulting rupture and destruction of eyeball \\
\hline 78 & T26.3 & Burn of other parts of eye and adnexa \\
\hline 79 & T26.6 & Corrosion of cornea and conjunctival sac \\
\hline 80 & T26.9 & Corrosion of eye and adnexa, part unspecified \\
\hline 81 & $\mathrm{~T} 27.1$ & Burn involving larynx and trachea with lung \\
\hline 82 & T27.5 & Corrosion involving larynx and trachea with lung \\
\hline 83 & T29.7 & Corrosion of multiple regions, at least one corrosion of third degree mentioned \\
\hline 84 & T31.0 & Burns involving less than $10 \%$ of body surface \\
\hline 85 & $\mathrm{~T} 31.2$ & Burns involving 20 to $29 \%$ of body surface \\
\hline 86 & T33.0 & Superficial frostbite of head \\
\hline 87 & T33.6 & Superficial frostbite of hip and thigh \\
\hline 88 & T33.8 & Superficial frostbite of ankle and foot \\
\hline 89 & T37.1 & Antimycobacterial drugs \\
\hline 90 & T39.0 & Salicylate \\
\hline 91 & $\mathrm{~T} 40.3$ & Methadone \\
\hline 92 & T41.5 & Therapeutic gases \\
\hline 93 & $\mathrm{~T} 42.2$ & Succinimide and oxazolidinedione \\
\hline 94 & T42.7 & Antiepileptic and sedative-hypnotis drugs, unspecified \\
\hline 95 & T44.8 & Centrally acting and adrenergic-neuron-blocking agents, NEC \\
\hline 96 & T45.5 & Anticoagulants \\
\hline 97 & T46.8 & Antivaricose drugs, including sclerosing agents \\
\hline 98 & T47.8 & Other agents primarily affecting the gastrointestinal system \\
\hline 99 & T50.7 & Analeptics and opioid receptor antagonists \\
\hline 100 & T51.8 & Other alcohols \\
\hline 101 & T53.0 & Carbon tetrachloride \\
\hline 102 & T53.3 & Tetrachloroethylene \\
\hline
\end{tabular}

(Continued to the next page) 
Appendix 1. Continued

\begin{tabular}{|c|c|c|}
\hline No. & ICD-10 & Diagnosis \\
\hline 103 & T56.0 & Lead and its compounds \\
\hline 104 & T59.8 & Other specified gases, fumes and vapours \\
\hline 105 & T60.2 & Other insecticides \\
\hline 106 & T63.0 & Snake venom \\
\hline 107 & T63.2 & Venom of scorpion \\
\hline 108 & T63.9 & Toxic effect of contact with unspecified venomous animal \\
\hline 109 & T65.9 & Toxic effect of unspecified substance \\
\hline 110 & T68.0 & Hypothermia \\
\hline 111 & T71.0 & Asphyxiation \\
\hline 112 & T73.8 & Other effects of deprivation \\
\hline 113 & T75.1 & Drowning and nonfatal submersion \\
\hline 114 & T79.5 & Traumatic anuria \\
\hline 115 & T80.9 & Unspecified complications following infusion, transfusion and therapeutic injection \\
\hline 116 & T82.3 & Mechanical complication of other vascular grafts \\
\hline 117 & T82.9 & Unspecified complication of cardiac and vascular prosthetic devices, implants and grafts \\
\hline 118 & T83.0 & Mechanical complication of urinary (indselling) catheter \\
\hline 119 & T83.8 & Other complications of genitourinary prosthetic devices, implants and grafts \\
\hline 120 & T87.4 & Infection of amputation stump \\
\hline 121 & T87.6 & Other and unspecified complications of amputation stump \\
\hline 122 & T90.9 & Sequelae of unspecified injury of head \\
\hline 123 & T91.9 & Sequelae of unspecified injury of neck and trunk \\
\hline 124 & T92.9 & Sequelae of unspecified injury of upper limb \\
\hline 125 & T94.0 & Sequelae of injuries involving multiple body regions \\
\hline 126 & T95.0 & Sequelae of burn, corrosion and frostbite of head and neck \\
\hline 127 & T95.1 & Sequelae of burn, corrosion and frostbite of trunk \\
\hline 128 & T95.9 & Sequelae of unspecified burn, corrosion and frostbite \\
\hline 129 & T98.1 & Sequelae of other and unspecified effects of external causes \\
\hline 130 & T98.3 & Sequelae of complications of surgical and medical care, NEC \\
\hline
\end{tabular}

ICD-10, International Classification of Diseases 10th edition; NEC, not elsewhere classified. 
Appendix 2. The value estimation methods used in the calculation of GBD-DALY and ICD-DALY

\begin{tabular}{|c|c|c|c|c|c|}
\hline Value & a (YLL) & a (YLD) & L (YLL) & $L(Y L D)$ & DW \\
\hline Definition & Age at death & Age at injury & Life expectancy & Disability duration & Disability weight \\
\hline GBD-DALY & DisMod II & DisMod II & Cohort absolute life table & DisMod II & 32 Injury nature \\
\hline ICD-DALY & Injury data base & Injury data base & Cohort absolute life table & Cohort absolute life table & 1,284 ICD S/T codes \\
\hline
\end{tabular}

GBD, Global Burden of Disease Study; DALY, disability-adjusted life year; ICD, International Classification of Disease 10th edition; YLL, years of life lost; YLD, years of life disabled; DW, disability weight. 
Yu Jin Kim, et al.

Appendix 3. Disability weight according to injury related ICD-10 codes

\begin{tabular}{|c|c|c|c|c|c|c|c|c|c|}
\hline ICD-10 & DW & ICD-10 & DW & ICD-10 & DW & ICD-10 & DW & ICD-10 & DW \\
\hline S00.0 & 0.02 & S38.3 & 0.7 & S83.7 & 0.3 & T25.4 & 0.3 & T54.2 & 0.29 \\
\hline S00.1 & 0.02 & S39.0 & 0.36 & S84.0 & 0.775 & T25.5 & 0.2 & T54.3 & 0.325 \\
\hline S00.2 & 0.01 & S39.6 & 0.7 & S84.1 & 0.3 & T25.6 & 0.3 & T54.9 & 0.325 \\
\hline S00.3 & 0.02 & S39.7 & 0.65 & S84.2 & 0.12 & $\mathrm{~T} 25.7$ & 0.375 & T55.0 & 0.08 \\
\hline S00.4 & 0.02 & S39.8 & 0.6 & S84.7 & 0.44 & T26.0 & 0.15 & T56.0 & 0.245 \\
\hline S00.5 & 0.005 & S39.9 & 0.6 & S84.8 & 0.4 & T26.1 & 0.18 & T56.1 & 0.2 \\
\hline S00.7 & 0.02 & S40.0 & 0.105 & S84.9 & 0.13 & T26.2 & 0.606 & T56.2 & 0.325 \\
\hline S00.8 & 0.02 & S40.7 & 0.048 & S85.0 & 0.45 & T26.3 & 0.129 & T56.3 & 0.325 \\
\hline S00.9 & 0.01 & S40.8 & 0.11 & S85.1 & 0.35 & T26.4 & 0.38 & T56.4 & 0.275 \\
\hline S01.0 & 0.12 & S40.9 & 0.05 & S85.2 & 0.5 & T26.5 & 0.39 & T56.5 & 0.15 \\
\hline S01.1 & 0.13 & S41.0 & 0.3 & S85.3 & 0.25 & T26.6 & 0.444 & T56.6 & 0.2 \\
\hline S01.2 & 0.125 & S41.1 & 0.305 & S85.4 & 0.21 & T26.7 & 0.5 & T56.8 & 0.2 \\
\hline S01.3 & 0.048 & S41.7 & 0.33 & S85.5 & 0.425 & T26.8 & 0.4 & T56.9 & 0.15 \\
\hline S01.4 & 0.05 & S41.8 & 0.12 & S85.7 & 0.4 & T26.9 & 0.258 & T57.0 & 0.15 \\
\hline S01.5 & 0.05 & S42.0 & 0.25 & S85.8 & 0.2 & T27.0 & 0.8 & T57.1 & 0.2 \\
\hline S01.7 & 0.18 & S42.1 & 0.23 & S85.9 & 0.25 & T27.1 & 0.697 & T57.2 & 0.2 \\
\hline S01.8 & 0.05 & S42.2 & 0.23 & S86.0 & 0.35 & T27.2 & 0.6 & T57.3 & 0.5 \\
\hline S01.9 & 0.04 & S42.3 & 0.23 & S86.1 & 0.39 & T27.3 & 0.4 & T57.8 & 0.275 \\
\hline S02.0 & 0.167 & S42.4 & 0.435 & S86.2 & 0.42 & T27.4 & 0.84 & T57.9 & 0.25 \\
\hline S02.1 & 0.4 & S42.7 & 0.25 & S86.3 & 0.18 & T27.5 & 1 & T58.0 & 0.45 \\
\hline S02.2 & 0.15 & S42.8 & 0.2 & S86.7 & 0.3 & T27.6 & 0.5 & T59.0 & 0.25 \\
\hline S02.3 & 0.16 & S42.9 & 0.2 & S86.8 & 0.35 & T27.7 & 0.825 & T59.1 & 0.5 \\
\hline S02.4 & 0.167 & S43.0 & 0.18 & S86.9 & 0.3 & T28.0 & 0.3 & T59.2 & 0.33 \\
\hline S02.5 & 0.1 & S43.1 & 0.25 & S87.0 & 0.5 & T28.1 & 0.5 & T59.3 & 0.25 \\
\hline S02.6 & 0.4 & S43.2 & 0.21 & S87.8 & 0.57 & T28.2 & 0.4 & T59.4 & 0.31 \\
\hline S02.7 & 0.48 & S43.3 & 0.4 & S88.0 & 0.75 & T28.3 & 0.55 & T59.5 & 0.435 \\
\hline S02.8 & 0.258 & S43.4 & 0.31 & S88.1 & 0.75 & T28.4 & 0.7 & T59.6 & 0.33 \\
\hline S02.9 & 0.3 & S43.5 & 0.11 & S88.9 & 0.7 & T28.5 & 0.35 & T59.7 & 0.2 \\
\hline S03.0 & 0.1 & S43.6 & 0.1 & S89.0 & 0.35 & T28.6 & 0.5 & T59.8 & 0.333 \\
\hline S03.1 & 0.15 & S43.7 & 0.09 & S89.7 & 0.45 & T28.7 & 0.55 & T59.9 & 0.4 \\
\hline S03.2 & 0.1 & S44.0 & 0.47 & S89.8 & 0.3 & T28.8 & 0.55 & T60.0 & 0.475 \\
\hline S03.3 & 0.08 & S44.1 & 0.475 & S89.9 & 0.091 & T28.9 & 0.55 & T60.1 & 0.25 \\
\hline S03.4 & 0.075 & S44.2 & 0.47 & S90.0 & 0.125 & T29.0 & 0.25 & T60.2 & 0.35 \\
\hline S03.5 & 0.06 & S44.3 & 0.28 & S90.1 & 0.055 & T29.1 & 0.17 & T60.3 & 0.8 \\
\hline S04.0 & 0.8 & S44.4 & 0.425 & S90.2 & 0.07 & T29.2 & 0.26 & T60.4 & 0.25 \\
\hline S04.1 & 0.2 & S44.5 & 0.1 & S90.3 & 0.05 & T29.3 & 0.6 & T60.8 & 0.6 \\
\hline S04.2 & 0.775 & S44.7 & 0.45 & S90.7 & 0.11 & T29.4 & 0.3 & T60.9 & 0.7 \\
\hline S04.3 & 0.75 & S44.8 & 0.41 & S90.8 & 0.115 & T29.5 & 0.12 & T61.0 & 0.2 \\
\hline S04.4 & 0.775 & S44.9 & 0.28 & S90.9 & 0.115 & T29.6 & 0.28 & T61.1 & 0.15 \\
\hline S04.5 & 0.765 & S45.0 & 0.35 & S91.0 & 0.215 & T29.7 & 0.545 & T61.2 & 0.3 \\
\hline S04.6 & 0.4 & S45.1 & 0.25 & S91.1 & 0.215 & T30.0 & 0.3 & T61.8 & 0.25 \\
\hline S04.7 & 0.25 & S45.2 & 0.25 & S91.2 & 0.12 & T30.1 & 0.12 & T61.9 & 0.25 \\
\hline S04.8 & 0.76 & S45.3 & 0.2 & S91.3 & 0.09 & T30.2 & 0.25 & T62.0 & 0.4 \\
\hline S04.9 & 0.3 & S45.7 & 0.2 & S91.7 & 0.215 & T30.3 & 0.4 & T62.1 & 0.15 \\
\hline S05.0 & 0.282 & S45.8 & 0.33 & S92.0 & 0.32 & T30.4 & 0.3 & T62.2 & 0.2 \\
\hline S05.1 & 0.325 & S45.9 & 0.33 & S92.1 & 0.4 & T30.5 & 0.3 & T62.8 & 0.15 \\
\hline S05.2 & 0.8 & S46.0 & 0.18 & S92.2 & 0.3 & T30.6 & 0.35 & T62.9 & 0.1 \\
\hline S05.3 & 0.45 & S46.1 & 0.444 & S92.3 & 0.3 & T30.7 & 0.4 & T63.0 & 0.35 \\
\hline S05.4 & 0.825 & S46.2 & 0.3 & S92.4 & 0.25 & T31.0 & 0.091 & T63.1 & 0.2 \\
\hline S05.5 & 0.998 & S46.3 & 0.25 & S92.5 & 0.333 & T31.1 & 0.2 & T63.2 & 0.231 \\
\hline S05.6 & 0.55 & S46.7 & 0.14 & S92.7 & 0.415 & T31.2 & 0.272 & T63.3 & 0.3 \\
\hline
\end{tabular}


Appendix 3. Continued

\begin{tabular}{|c|c|c|c|c|c|c|c|c|c|}
\hline ICD-10 & DW & ICD-10 & DW & ICD-10 & DW & ICD-10 & DW & ICD-10 & DW \\
\hline S05.7 & 0.8 & S46.8 & 0.275 & S92.9 & 0.405 & T31.3 & 0.4 & T63.4 & 0.05 \\
\hline S05.8 & 0.3 & S46.9 & 0.275 & S93.0 & 0.2 & T31.4 & 0.5 & T63.5 & 0.12 \\
\hline S06.0 & 0.2 & S48.0 & 0.45 & S93.2 & 0.25 & T31.6 & 0.65 & T63.8 & 0.3 \\
\hline S06.1 & 0.61 & S48.1 & 0.45 & S93.3 & 0.286 & T31.7 & 0.8 & T63.9 & 0.029 \\
\hline S06.2 & 0.6 & S48.9 & 0.45 & S93.4 & 0.165 & T31.8 & 0.775 & T64.0 & 0.36 \\
\hline S06.4 & 0.4 & S49.7 & 0.3 & S93.6 & 0.07 & T32.0 & 0.3 & T65.1 & 0.4 \\
\hline S06.5 & 0.71 & S49.8 & 0.27 & S94.0 & 0.3 & T32.1 & 0.35 & T65.2 & 0.3 \\
\hline S06.6 & 0.57 & S49.9 & 0.25 & S94.1 & 0.3 & T32.2 & 0.45 & T65.3 & 0.4 \\
\hline S06.7 & 0.8 & S50.0 & 0.15 & S94.2 & 0.17 & T32.3 & 0.55 & T65.4 & 0.26 \\
\hline S06.8 & 0.585 & S50.1 & 0.08 & S94.3 & 0.25 & T32.4 & 0.6 & T65.5 & 0.16 \\
\hline S06.9 & 0.6 & S50.7 & 0.05 & S94.7 & 0.4 & T32.5 & 0.65 & T65.6 & 0.14 \\
\hline S07.9 & 0.72 & S51.7 & 0.09 & S95.1 & 0.31 & T32.9 & 0.95 & T67.0 & 0.25 \\
\hline S08.0 & 0.425 & S51.8 & 0.1 & S95.2 & 0.12 & T33.0 & 0.091 & T67.1 & 0.275 \\
\hline S08.1 & 0.575 & S51.9 & 0.1 & S95.7 & 0.259 & T33.1 & 0.05 & T67.2 & 0.2 \\
\hline S08.8 & 0.565 & S52.0 & 0.35 & S95.8 & 0.15 & T33.2 & 0.16 & T67.3 & 0.215 \\
\hline S08.9 & 0.535 & S52.1 & 0.35 & S95.9 & 0.31 & T33.3 & 0.06 & T67.4 & 0.215 \\
\hline S09.0 & 0.2 & S52.2 & 0.38 & S96.0 & 0.3 & T33.4 & 0.05 & T67.5 & 0.15 \\
\hline S09.1 & 0.1 & S52.3 & 0.38 & S96.1 & 0.18 & T33.5 & 0.06 & T67.6 & 0.11 \\
\hline S09.2 & 0.2 & S52.4 & 0.4 & S96.2 & 0.3 & T33.6 & 0.029 & T67.7 & 0.24 \\
\hline S09.7 & 0.45 & S52.5 & 0.38 & S96.7 & 0.3 & T33.7 & 0.075 & T67.8 & 0.13 \\
\hline S09.8 & 0.16 & S52.6 & 0.4 & S96.8 & 0.3 & T33.8 & 0.048 & T67.9 & 0.15 \\
\hline S11.1 & 0.3 & S53.4 & 0.07 & S98.3 & 0.57 & T34.6 & 0.3 & T70.2 & 0.15 \\
\hline S11.2 & 0.3 & S54.0 & 0.167 & S98.4 & 0.55 & T34.7 & 0.55 & T70.3 & 0.26 \\
\hline S11.7 & 0.35 & S54.1 & 0.42 & S99.0 & 0.25 & T34.8 & 0.53 & T70.4 & 0.225 \\
\hline S11.8 & 0.35 & S54.2 & 0.375 & S99.7 & 0.23 & T34.9 & 0.575 & T70.8 & 0.2 \\
\hline S11.9 & 0.3 & S54.3 & 0.36 & S99.8 & 0.12 & T35.0 & 0.05 & T70.9 & 0.25 \\
\hline S12.0 & 0.9 & S54.7 & 0.4 & S99.9 & 0.18 & T35.1 & 0.7 & T71.0 & 0.9 \\
\hline S12.1 & 0.8 & S54.8 & 0.4 & T00.0 & 0.1 & T35.2 & 0.15 & T73.0 & 0.05 \\
\hline $\mathrm{S} 12.2$ & 0.6 & S54.9 & 0.375 & T00.1 & 0.06 & T35.3 & 0.14 & T73.1 & 0.08 \\
\hline S12.7 & 0.71 & S55.0 & 0.36 & T00.2 & 0.04 & T35.4 & 0.6 & T73.2 & 0.1 \\
\hline S12.8 & 0.4 & S55.1 & 0.17 & T00.3 & 0.06 & T35.5 & 0.15 & T73.3 & 0.11 \\
\hline S12.9 & 0.6 & S55.2 & 0.17 & T00.6 & 0.07 & T35.6 & 0.15 & T73.8 & 0.129 \\
\hline S13.0 & 0.6 & S55.7 & 0.1 & T00.8 & 0.08 & T35.7 & 0.12 & T73.9 & 0.15 \\
\hline S13.1 & 0.7 & S55.8 & 0.15 & T00.9 & 0.07 & T36.0 & 0.1 & T74.0 & 0.2 \\
\hline S13.2 & 0.65 & S55.9 & 0.36 & T01.0 & 0.22 & T36.1 & 0.1 & T74.1 & 0.25 \\
\hline S13.3 & 0.9 & S56.0 & 0.4 & T01.1 & 0.31 & T36.2 & 0.4 & T74.2 & 0.25 \\
\hline S13.4 & 0.15 & S56.1 & 0.2 & T01.2 & 0.25 & T36.3 & 0.1 & T74.3 & 0.25 \\
\hline S13.5 & 0.175 & S56.2 & 0.708 & T01.3 & 0.15 & T36.4 & 0.25 & T74.8 & 0.25 \\
\hline S13.6 & 0.26 & S56.3 & 0.3 & T01.6 & 0.303 & T36.5 & 0.125 & T74.9 & 0.25 \\
\hline
\end{tabular}

(Continued to the next page) 
Yu Jin Kim, et al.

Appendix 3. Continued

\begin{tabular}{|c|c|c|c|c|c|c|c|c|c|}
\hline ICD-10 & DW & ICD-10 & DW & ICD-10 & DW & ICD-10 & DW & ICD-10 & DW \\
\hline S14.0 & 0.8 & S56.4 & 0.3 & T01.8 & 0.325 & T36.6 & 0.125 & T75.0 & 0.7 \\
\hline S14.1 & 0.5 & S56.5 & 0.2 & T01.9 & 0.45 & T36.7 & 0.175 & T75.1 & 0.857 \\
\hline S14.2 & 0.6 & S56.7 & 0.3 & T02.0 & 0.5 & T36.8 & 0.175 & T75.2 & 0.31 \\
\hline S14.3 & 0.667 & S56.8 & 0.3 & T02.1 & 0.65 & T36.9 & 0.1 & T75.3 & 0.03 \\
\hline S14.4 & 0.048 & S57.0 & 0.3 & T02.2 & 0.45 & T37.0 & 0.25 & T75.4 & 0.25 \\
\hline S14.5 & 0.55 & S57.8 & 0.5 & T02.3 & 0.45 & T37.1 & 0.5 & T75.8 & 0.16 \\
\hline S14.6 & 0.35 & S57.9 & 0.48 & T02.4 & 0.65 & T37.2 & 0.15 & T78.0 & 0.5 \\
\hline S15.0 & 0.7 & S58.0 & 0.735 & T02.5 & 0.65 & T37.3 & 0.1 & T78.1 & 0.15 \\
\hline S15.1 & 0.5 & S58.1 & 0.73 & T02.6 & 0.68 & T37.4 & 0.1 & T78.2 & 0.625 \\
\hline S15.2 & 0.4 & S58.9 & 0.725 & T02.7 & 0.75 & T37.5 & 0.2 & T78.3 & 0.3 \\
\hline S15.3 & 0.5 & S59.0 & 0.37 & T02.8 & 0.7 & T37.8 & 0.125 & T78.4 & 0.185 \\
\hline S15.7 & 0.6 & S59.7 & 0.36 & T02.9 & 0.7 & T37.9 & 0.12 & T78.8 & 0.1 \\
\hline S15.8 & 0.3 & S59.8 & 0.4 & T03.0 & 0.5 & T38.0 & 0.25 & T78.9 & 0.2 \\
\hline S15.9 & 0.444 & S59.9 & 0.17 & T03.1 & 0.2 & T38.1 & 0.12 & T79.0 & 0.675 \\
\hline S16.0 & 0.3 & S60.0 & 0.048 & T03.2 & 0.1 & T38.2 & 0.225 & T79.1 & 0.5 \\
\hline S17.0 & 0.575 & S60.1 & 0.085 & T03.3 & 0.3 & T38.3 & 0.275 & T79.2 & 0.325 \\
\hline S17.8 & 0.4 & S60.2 & 0.1 & T03.4 & 0.3 & T38.4 & 0.15 & T79.3 & 0.265 \\
\hline S17.9 & 0.4 & S60.7 & 0.05 & T03.8 & 0.1 & T38.5 & 0.1 & T79.4 & 0.5 \\
\hline S18.0 & 0.95 & S60.8 & 0.3 & T03.9 & 0.3 & T38.6 & 0.125 & T79.5 & 0.9 \\
\hline S19.0 & 0.43 & S60.9 & 0.06 & T04.0 & 0.68 & T38.7 & 0.2 & T79.6 & 0.29 \\
\hline S19.7 & 0.45 & S61.0 & 0.15 & T04.1 & 0.6 & T38.8 & 0.15 & T79.7 & 0.3 \\
\hline S19.8 & 0.36 & S61.1 & 0.1 & T04.2 & 0.58 & T38.9 & 0.15 & T79.8 & 0.35 \\
\hline S19.9 & 0.5 & S61.7 & 0.1 & T04.3 & 0.756 & T39.0 & 0.25 & T79.9 & 0.32 \\
\hline S20.0 & 0.029 & S61.8 & 0.2 & T04.4 & 0.625 & T39.1 & 0.25 & T80.0 & 0.32 \\
\hline S20.1 & 0.03 & S61.9 & 0.1 & T04.7 & 0.65 & T39.2 & 0.2 & T80.1 & 0.34 \\
\hline S20.2 & 0.03 & S62.0 & 0.2 & T04.8 & 0.575 & T39.3 & 0.07 & T80.2 & 0.2 \\
\hline S20.3 & 0.03 & S62.1 & 0.2 & T04.9 & 0.615 & T39.4 & 0.15 & T80.3 & 0.525 \\
\hline S20.4 & 0.029 & S62.2 & 0.15 & T05.0 & 0.85 & T39.8 & 0.25 & T80.4 & 0.31 \\
\hline S20.7 & 0.03 & S62.3 & 0.18 & T05.1 & 0.89 & T39.9 & 0.2 & T80.5 & 0.5 \\
\hline S20.8 & 0.017 & S62.4 & 0.2 & T05.2 & 0.9 & T40.0 & 0.15 & T80.6 & 0.475 \\
\hline S21.0 & 0.4 & S62.5 & 0.2 & T05.3 & 0.75 & T40.1 & 0.16 & T80.8 & 0.3 \\
\hline S21.1 & 0.15 & S62.6 & 0.285 & T05.4 & 0.78 & $\mathrm{~T} 40.2$ & 0.15 & T80.9 & 0.167 \\
\hline S21.2 & 0.375 & S62.7 & 0.28 & T05.5 & 0.82 & T40.3 & 0.111 & T81.0 & 0.275 \\
\hline S21.7 & 0.3 & S62.8 & 0.32 & T05.6 & 0.796 & T40.4 & 0.3 & T81.1 & 0.6 \\
\hline S21.8 & 0.38 & S63.0 & 0.2 & T05.8 & 0.875 & T40.5 & 0.515 & T81.2 & 0.17 \\
\hline S21.9 & 0.18 & S63.1 & 0.333 & T05.9 & 0.9 & T40.6 & 0.22 & T81.3 & 0.5 \\
\hline S22.0 & 0.45 & S63.2 & 0.286 & T06.0 & 0.85 & T40.7 & 0.375 & T81.4 & 0.2 \\
\hline S22.1 & 0.575 & S63.3 & 0.225 & T06.1 & 0.775 & T40.8 & 0.4 & T81.5 & 0.525 \\
\hline S22.2 & 0.34 & S63.4 & 0.22 & T06.2 & 0.5 & T40.9 & 0.15 & T81.6 & 0.6 \\
\hline S22.3 & 0.2 & S63.5 & 0.07 & T06.3 & 0.444 & T41.0 & 0.2 & T81.7 & 0.22 \\
\hline S22.4 & 0.5 & S63.6 & 0.115 & T06.4 & 0.3 & T41.1 & 0.7 & T81.8 & 0.2 \\
\hline S22.5 & 0.63 & S63.7 & 0.091 & T06.5 & 0.6 & T41.2 & 0.7 & T81.9 & 0.525 \\
\hline S22.8 & 0.333 & S64.0 & 0.19 & T06.8 & 0.22 & T41.3 & 0.15 & T82.0 & 0.5 \\
\hline S22.9 & 0.4 & S64.1 & 0.225 & T07.0 & 0.3 & T41.4 & 0.225 & T82.1 & 0.485 \\
\hline S23.0 & 0.27 & S64.2 & 0.36 & T08.0 & 0.725 & T41.5 & 0.048 & T82.2 & 0.7 \\
\hline S23.1 & 0.5 & S64.3 & 0.3 & T09.0 & 0.007 & T42.0 & 0.16 & T82.3 & 0.65 \\
\hline S23.2 & 0.38 & S64.4 & 0.18 & T09.1 & 0.19 & T42.1 & 0.28 & T82.4 & 0.3 \\
\hline S23.3 & 0.091 & S64.7 & 0.3 & T09.2 & 0.25 & $\mathrm{~T} 42.2$ & 0.091 & T82.5 & 0.675 \\
\hline S23.4 & 0.13 & S64.8 & 0.3 & T09.3 & 0.37 & T42.3 & 0.25 & T82.6 & 0.675 \\
\hline S23.5 & 0.2 & S64.9 & 0.2 & T09.4 & 0.725 & T42.4 & 0.2 & T82.7 & 0.675 \\
\hline S24.0 & 0.7 & S65.0 & 0.25 & T09.5 & 0.2 & T42.5 & 0.25 & T82.8 & 0.54 \\
\hline
\end{tabular}


Appendix 3. Continued

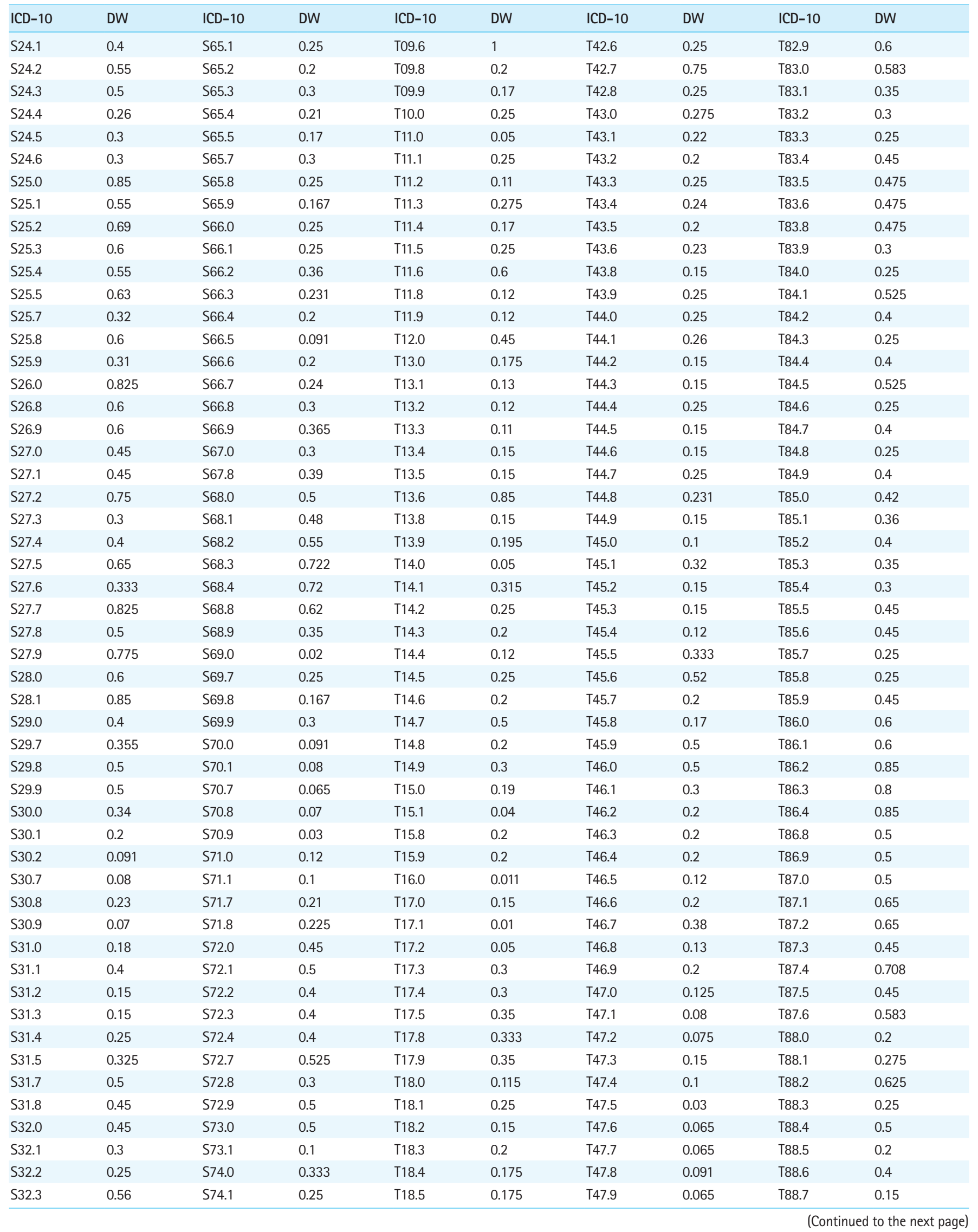


Yu Jin Kim, et al.

Appendix 3. Continued

\begin{tabular}{|c|c|c|c|c|c|c|c|c|c|}
\hline ICD-10 & DW & ICD-10 & DW & ICD-10 & DW & ICD-10 & DW & ICD-10 & DW \\
\hline S32.4 & 0.32 & S74.2 & 0.2 & T18.8 & 0.21 & T48.0 & 0.125 & T88.8 & 0.475 \\
\hline S32.5 & 0.52 & S74.7 & 0.38 & T18.9 & 0.2 & T48.1 & 0.25 & T88.9 & 0.4 \\
\hline S32.7 & 0.6 & S74.8 & 0.36 & T19.0 & 0.315 & T48.2 & 0.12 & T90.0 & 0.1 \\
\hline S32.8 & 0.48 & S74.9 & 0.19 & T19.1 & 0.2 & T48.3 & 0.15 & T90.1 & 0.39 \\
\hline S33.0 & 0.456 & S75.0 & 0.475 & T19.2 & 0.07 & T48.4 & 0.1 & T90.2 & 0.25 \\
\hline S33.1 & 0.685 & S75.1 & 0.45 & T19.3 & 0.3 & T48.5 & 0.06 & T90.3 & 0.5 \\
\hline S33.2 & 0.3 & S75.2 & 0.583 & T19.8 & 0.1 & T48.6 & 0.1 & T90.4 & 0.3 \\
\hline S33.3 & 0.5 & S75.7 & 0.465 & T19.9 & 0.18 & T48.7 & 0.13 & T90.5 & 0.55 \\
\hline S33.4 & 0.34 & S75.8 & 0.46 & T20.0 & 0.3 & T49.0 & 0.1 & T90.8 & 0.3 \\
\hline S33.5 & 0.225 & S75.9 & 0.4 & T20.1 & 0.048 & T49.1 & 0.08 & T90.9 & 0.444 \\
\hline S33.6 & 0.15 & S76.0 & 0.46 & T20..2 & 0.3 & T49.2 & 0.05 & T91.0 & 0.1 \\
\hline S33.7 & 0.1 & S76.1 & 0.45 & T20.3 & 0.625 & T49.3 & 0.05 & T91.1 & 0.55 \\
\hline S34.0 & 0.65 & S76.2 & 0.48 & T20.4 & 0.5 & T49.4 & 0.1 & T91.2 & 0.39 \\
\hline S34.1 & 0.35 & S76.3 & 0.25 & T20.5 & 0.12 & T49.5 & 0.05 & T91.3 & 0.41 \\
\hline S34.2 & 0.52 & S76.4 & 0.2 & T20.6 & 0.25 & T49.6 & 0.1 & T91.4 & 0.6 \\
\hline S34.3 & 0.39 & S76.7 & 0.3 & T20.7 & 0.52 & T49.7 & 0.1 & T91.5 & 0.3 \\
\hline S34.4 & 0.57 & S77.0 & 0.6 & T21.0 & 0.25 & T49.8 & 0.1 & T91.8 & 0.4 \\
\hline S34.5 & 0.3 & S77.1 & 0.58 & $\mathrm{~T} 21.1$ & 0.15 & T49.9 & 0.1 & T91.9 & 0.333 \\
\hline S34.6 & 0.23 & S77.2 & 0.65 & T21.2 & 0.315 & T50.0 & 0.1 & T92.0 & 0.21 \\
\hline S34.8 & 0.231 & S78.0 & 0.8 & T21.3 & 0.333 & T50.1 & 0.1 & T92.1 & 0.25 \\
\hline S35.0 & 0.825 & S78.1 & 0.77 & T21.4 & 0.35 & T50.2 & 0.18 & T92.2 & 0.25 \\
\hline S35.1 & 0.6 & S78.9 & 0.75 & $\mathrm{~T} 21.5$ & 0.23 & T50.3 & 0.1 & T92.3 & 0.12 \\
\hline S35.2 & 0.73 & S79.0 & 0.3 & T21.6 & 0.33 & T50.4 & 0.15 & T92.4 & 0.25 \\
\hline S35.3 & 0.785 & S79.7 & 0.35 & T21.7 & 0.5 & T50.5 & 0.1 & T92.5 & 0.26 \\
\hline S35.4 & 0.5 & S79.8 & 0.15 & T22.0 & 0.15 & T50.6 & 0.12 & T92.6 & 0.39 \\
\hline S35.5 & 0.49 & S79.9 & 0.485 & T22.1 & 0.05 & T50.7 & 0.149 & T92.8 & 0.12 \\
\hline S35.7 & 0.6 & S80.0 & 0.09 & T22.2 & 0.2 & T50.8 & 0.18 & T92.9 & 0.333 \\
\hline S35.8 & 0.575 & S80.1 & 0.12 & T22.3 & 0.46 & T50.9 & 0.28 & T93.0 & 0.13 \\
\hline S35.9 & 0.575 & S80.7 & 0.06 & T22.4 & 0.4 & T51.0 & 0.12 & T93.1 & 0.25 \\
\hline S36.0 & 0.48 & S80.8 & 0.06 & T22.5 & 0.1 & T51.1 & 0.65 & T93.2 & 0.15 \\
\hline S36.1 & 0.635 & S80.9 & 0.006 & T22.6 & 0.275 & T51.2 & 0.25 & T93.3 & 0.12 \\
\hline S36.2 & 0.55 & S81.0 & 0.1 & T22.7 & 0.35 & T51.3 & 0.25 & T93.4 & 0.36 \\
\hline S36.3 & 0.665 & S81.7 & 0.24 & T23.0 & 0.15 & T51.8 & 0.212 & T93.5 & 0.12 \\
\hline S36.4 & 0.55 & S81.8 & 0.12 & T23.1 & 0.05 & T51.9 & 0.22 & T93.6 & 0.4 \\
\hline S36.5 & 0.68 & S81.9 & 0.21 & T23.2 & 0.16 & T52.0 & 0.3 & T93.8 & 0.25 \\
\hline S36.6 & 0.685 & S82.0 & 0.3 & T23.3 & 0.4 & T52.1 & 0.2 & T93.9 & 0.3 \\
\hline S36.7 & 0.65 & S82.1 & 0.3 & T23.4 & 0.275 & T52.2 & 0.27 & T94.0 & 0.393 \\
\hline S36.8 & 0.53 & S82.2 & 0.49 & T23.5 & 0.2 & T52.3 & 0.29 & T94.1 & 0.2 \\
\hline S36.9 & 0.6 & S82.3 & 0.667 & T23.6 & 0.3 & T52.4 & 0.25 & T95.0 & 0.545 \\
\hline S37.0 & 0.55 & S82.4 & 0.2 & T23.7 & 0.425 & T52.8 & 0.315 & T95.1 & 0.32 \\
\hline S37.1 & 0.685 & S82.5 & 0.2 & T24.0 & 0.24 & T52.9 & 0.3 & T95.2 & 0.3 \\
\hline S37.2 & 0.68 & S82.6 & 0.45 & T24.1 & 0.12 & T53.0 & 0.35 & T95.3 & 0.2 \\
\hline S37.3 & 0.625 & S82.7 & 0.5 & T24.2 & 0.3 & T53.1 & 0.25 & T95.4 & 0.25 \\
\hline S37.4 & 0.625 & S82.8 & 0.3 & T24.3 & 0.419 & T53.2 & 0.21 & T95.8 & 0.25 \\
\hline S37.5 & 0.5 & S82.9 & 0.35 & T24.4 & 0.4 & T53.3 & 0.048 & T95.9 & 0.31 \\
\hline S37.6 & 0.45 & S83.0 & 0.41 & T24.5 & 0.1 & T53.4 & 0.21 & T96.0 & 0.16 \\
\hline S37.7 & 0.65 & S83.1 & 0.2 & T24.6 & 0.32 & T53.5 & 0.2 & T97.0 & 0.1 \\
\hline S37.8 & 0.52 & S83.2 & 0.667 & $\mathrm{~T} 24.7$ & 0.4 & T53.6 & 0.3 & T98.0 & 0.35 \\
\hline S37.9 & 0.55 & S83.3 & 0.375 & T25.0 & 0.2 & T53.7 & 0.15 & T98.1 & 0.667 \\
\hline S38.0 & 0.705 & S83.4 & 0.1 & T25.1 & 0.1 & T53.9 & 0.15 & T98.2 & 0.3 \\
\hline S38.1 & 0.7 & S83.5 & 0.1 & T25.2 & 0.3 & T54.0 & 0.2 & T98.3 & 0.5 \\
\hline S38.2 & 0.857 & S83.6 & 0.1 & T25.3 & 0.5 & T54.1 & 0.54 & & \\
\hline
\end{tabular}

ICD-10, International Classification of Disease 10th edition; DW, disability weight. 
Appendix 4. The excluded categories from the comparison with ICDDALY and GBD-DALY

\begin{tabular}{|c|c|}
\hline ICD code & Category or subcategory \\
\hline T14.2 & Fracture of unspecified body region \\
\hline T14.8 & Other injuries of unspecified body region \\
\hline T14.9 & Injury, unspecified \\
\hline Т34.0-Т34.9 & Frostbite with tissue necrosis \\
\hline T35.0-Т35.7 & Frostbite involving multiple body regions and unspecified frostbite \\
\hline T66 & Unspecified effects of radiation \\
\hline T67.0-T67.9 & Effects of heat and light \\
\hline T68 & Hypothermia \\
\hline T69.0-T69.9 & Other effects of reduced temperature \\
\hline T70.0-T70.9 & Effects of air pressure and water pressure \\
\hline T71 & Asphyxiation \\
\hline T73.0-T73.9 & Effects of other deprivation \\
\hline T74.0-T74.9 & Maltreatment syndromes \\
\hline T75.0-T75.8 & Effects of other external causes \\
\hline T78.0-T78.9 & Adverse effects, NEC \\
\hline T79.0-T79.9 & Certain early complications of trauma, NEC \\
\hline T80.0-T80.9 & Complications following infusion, transfusion and therapeutic injection \\
\hline T81.0-T81.9 & Complications of procedures, NEC \\
\hline T82.0-T82.9 & $\begin{array}{l}\text { Complications of cardiac and vascular prosthetic devices, implants } \\
\text { and grafts }\end{array}$ \\
\hline T83.0-T83.9 & $\begin{array}{l}\text { Complications of genitourinary prosthetic devices, implants and } \\
\text { grafts }\end{array}$ \\
\hline T84.0-T84.9 & $\begin{array}{l}\text { Complications of internal orthopaedic prosthetic devices, implants } \\
\text { and grafts }\end{array}$ \\
\hline T85.0-T85.9 & $\begin{array}{l}\text { Complications of other internal prosthetic devices, implants and } \\
\text { grafts }\end{array}$ \\
\hline T86.0-T86.9 & Failure and rejection of transplanted organs and tissues \\
\hline T87.0-Т87.6 & Complications peculiar to reattachment and amputation \\
\hline T88.0-T88.9 & Other complications of surgical and medical care, NEC \\
\hline T94.0,T94.1 & Sequelae of injuries involving multiple and unspecified body regions \\
\hline T95.0-T95.9 & Sequelae of burns, corrosions and frostbite \\
\hline T96 & $\begin{array}{l}\text { Sequelae of poisoning by drugs, medicaments and biological } \\
\text { substances }\end{array}$ \\
\hline T97 & $\begin{array}{l}\text { Sequelae of toxic effects of substances chiefly nonmedicinal as to } \\
\text { source }\end{array}$ \\
\hline T98.0-T98.3 & Sequelae of other and unspecified effects of external causes \\
\hline
\end{tabular}

ICD, International Classification of Disease 10th edition; DALY, disability-adjusted life year; GBD, Global Burden of Disease Study; NEC, not elsewhere classified. 